\title{
An approach to high-throughput $X$-ray diffraction analysis of combinatorial polycrystalline thin film libraries
}

\author{
S. Roncallo, O. Karimi, K. D. Rogers, D. W. Lane and S. A. Ansari
}

J. Appl. Cryst. (2009). 42, 174-178

Copyright (C) International Union of Crystallography

Author(s) of this paper may load this reprint on their own web site or institutional repository provided that this cover page is retained. Republication of this article or its storage in electronic databases other than as specified above is not permitted without prior permission in writing from the IUCr.

For further information see http://journals.iucr.org/services/authorrights.html

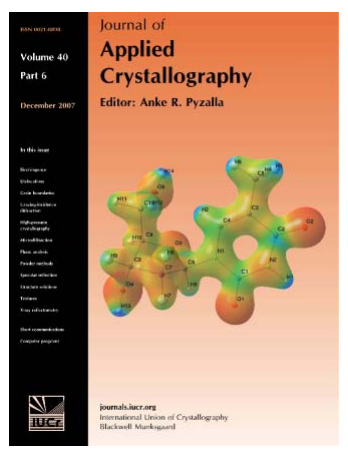

\begin{abstract}
Many research topics in condensed matter research, materials science and the life sciences make use of crystallographic methods to study crystalline and non-crystalline matter with neutrons, X-rays and electrons. Articles published in the Journal of Applied Crystallography focus on these methods and their use in identifying structural and diffusioncontrolled phase transformations, structure-property relationships, structural changes of defects, interfaces and surfaces, etc. Developments of instrumentation and crystallographic apparatus, theory and interpretation, numerical analysis and other related subjects are also covered. The journal is the primary place where crystallographic computer program information is published.
\end{abstract}

\section{Crystallography Journals Online is available from journals.iucr.org}


Journal of

Applied

Crystallography

ISSN 0021-8898

Received 1 August 2008

Accepted 26 January 2009

(C) 2009 International Union of Crystallography

Printed in Singapore - all rights reserved

\section{An approach to high-throughput $\mathrm{X}$-ray diffraction analysis of combinatorial polycrystalline thin film libraries}

\author{
S. Roncallo, ${ }^{*}$ O. Karimi, K. D. Rogers, D. W. Lane and S. A. Ansari \\ Cranfield University, Shrivenham Campus, Swindon, Wiltshire SN6 8LA, UK. Correspondence \\ e-mail: s.roncallo@cranfield.ac.uk
}

\begin{abstract}
With the demand for higher rates of discovery in the materials field, characterization techniques that are capable of rapidly and reliably surveying the characteristics of large numbers of samples are essential. A chemical combinatorial approach using thin films can provide detailed phase diagrams without the need to produce multiple, individual samples. This is achieved with compositional gradients forming high-density libraries. Conventional raster scanning of chemical or structural probes is subsequently used to interrogate the libraries. A new, alternative approach to raster scanning is introduced to provide a method of high-throughput data collection and analysis using an X-ray diffraction probe. Libraries are interrogated with an extended X-ray source and the scattering data collected using an area detector. A simple technique of 'partitioning' this scattering distribution enables determination of information comparable to conventional raster scanned results but in a dramatically reduced collection time. The technique has been tested using synthetic X-ray scattering distributions and those obtained from contrived samples. In all cases, the partitioning algorithm is shown to be robust and to provide reliable data; discrimination along the library principal axis is shown to be $\sim 500 \mu \mathrm{m}$ and the lattice parameter resolution to be $\sim 10^{-3} \AA \mathrm{mm}^{-1}$. The limitations of the technique are discussed and future potential applications described.
\end{abstract}

\section{Introduction}

Production and characterization of new functional materials is an important component of materials science advancement. However, the identification of new material compositions with desirable and optimized properties (e.g. through the preparation of phase diagrams) is traditionally time consuming and costly; less than $5 \%$ of ternary and $1 \%$ of quaternary alloys have been fully characterized (Zhao, 2006). A technology area that is currently seeking to rapidly survey a wide range of materials with substantial compositional variation is photovoltaics. The future successful development of solar cells requires the identification of new materials with properties optimized for features such as energy generation, long-term sustainability and low cost. Material optimization required for solar cell improvements includes, for example, optimization of the conductivity and optical properties of transparent conducting oxides such as $\mathrm{In}_{2} \mathrm{O}_{3}$ by the addition of elemental dopants (van Hest et al., 2006). The multitude of possible combinations of these additions means that a full systematic study of their effects by conventional methods is not economically feasible.

In recent years the use of combinatorial techniques has translated from organic (e.g. pharmaceutical) to inorganic materials. The exploitation of thin inorganic films fabricated into high-density chemical libraries has shown great potential for rapid surveying of wide compositional ranges (Takeuchi et al., 2006). Libraries with homogeneous compositional areas and continuous compositional spreads (CCSs) can now be produced routinely in a single deposition step (Thelakkat et al., 2004). High-throughput or combinatorial X-ray diffraction studies of polycrystalline thin films have so far relied on conventional raster scanning of either CCS samples (e.g. Gorrie et al., 2008) or arrays of individual samples (e.g. Yanase et al., 2002), with the high-throughput aims being achieved by the automation of this data collection method.

Previous work by Ohtani et al. (2001) links structural determination to spatial mapping for epitaxial samples, by the use of simultaneous data collection with a wide X-ray beam. However, this method has not previously been adopted for polycrystalline materials owing to difficulties arising from the interpretation of the overlapped diffraction data.

In this paper, a new approach is demonstrated which uses both an extended X-ray beam profile and analytical interpretation of subsequent scattering data from polycrystalline thin films. In this initial assessment, the new approach is shown to significantly increase the interrogation speed of X-ray diffraction data compared with conventional methods (such as pencil beam probes). 
The study investigates the potential use of a 'line' probe that illuminates a narrow rectangular area along a principal axis $(y)$ of the library. The two-dimensional scattering distribution is subsequently measured in a single exposure using an area detector. This scatter distribution is thus the superposition of diffraction patterns from all loci along the illuminated area. A significant challenge, as a result of the complexity of polycrystalline materials, is to derive the structural information corresponding to each locus from this scatter distribution, a process termed 'partitioning'. The whole library can subsequently be interrogated by scanning the sample in one direction through the X-ray line beam.

\section{Method}

\subsection{Partitioning}

The method used here was developed following consideration of several alternative approaches. These included deconvolution and wavelet synthesis which, although

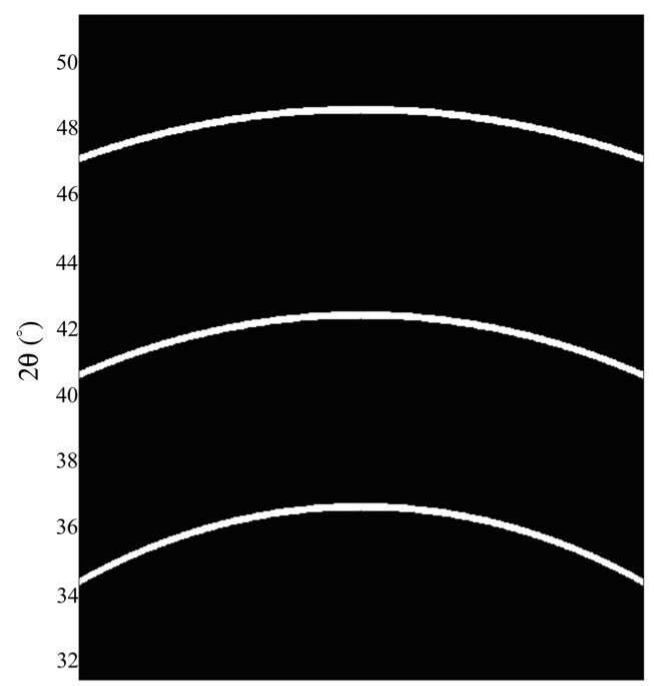

(a)

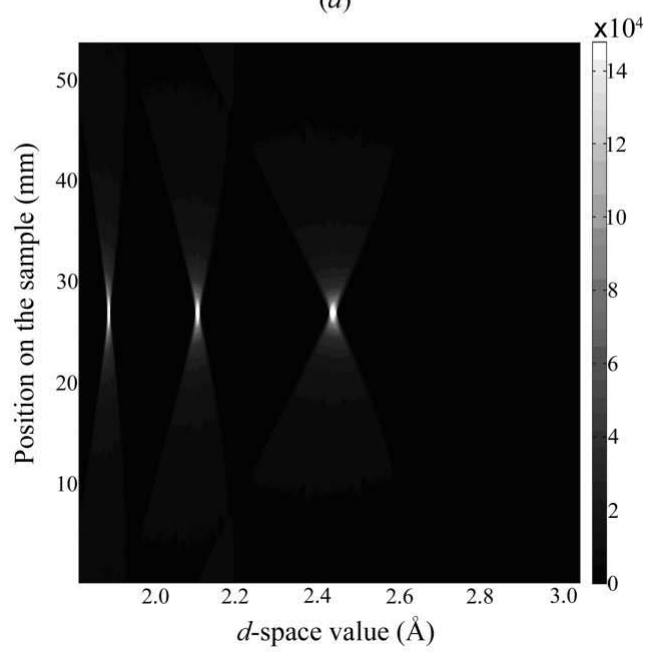

(b)

\section{Figure 1}

Simulated diffraction intensity distribution of an ideal single compositional area $(a)$ and the associated transformed distribution $(b)$. successful elsewhere, were undermined by the poor signal-tonoise ratio that is characteristic of these scattering distributions. Thus we have adopted a pragmatic, empirical approach that transforms the intensity scattering distribution data directly into a normalized intensity map $[\operatorname{In}(d, y)]$ on a $d$ spacing versus locus $(y)$ grid. From such maps, phase and lattice parameters at any point along the library can be determined. The transformation process is simply achieved through

$$
\operatorname{In}(d, y)=\int_{\chi_{1}}^{\chi_{2}} I_{2 \theta, y}(\chi) \mathrm{d} \chi /\left(\chi_{2}-\chi_{1}\right)
$$

where $\chi_{1}$ and $\chi_{2}$ are the azimuthal limits of the detector and are dependent on scattering angle for our system, and $I_{2 \theta, y}(\chi)$ is the measured intensity at particular values of $2 \theta$ and $y$.

\subsection{Experimental}

To illustrate this partitioning method and test its limitations, we have produced (i) a series of simulated scattering patterns and (ii) experimental scattering data from well controlled, 'phantom' samples. In all cases we have applied the partitioning algorithm to produce the corresponding $\operatorname{In}(d, y)$ maps and directly compared these with the simulation models and phantoms.

All the experimental scattering data were measured using a Bruker D8 diffractometer fitted with a GADDS detector. The incident X-ray beam $(\mathrm{Cu} K \alpha)$ was conditioned to produce a beam of $\sim 30 \times 1 \mathrm{~mm}$ at the sample using a single Geöbel mirror. Scattering data were corrected for detector distortions (geometric and response) prior to analysis.

The transformation algorithms were embodied within a new code, Bandit, that was developed in Matlab (The MathWorks Inc., Natick, MA, USA). This code reads the detector output and applies a calibration based upon an external standard to ensure accurate $d$-spacing determinations from each intensity maximum. It subsequently applies the transformation (1) and produces $\operatorname{In}(d, y)$ contour plots. Bandit was also used to produce the simulated scattering distributions based upon structural models that were varied along the length of the X-ray beam.

The phantom samples examined were produced from bulk powders and polycrystalline, metallic thin films, fabricated by sputter coating. These included the following:

(a) A glass slide containing a linear array of wells $(\sim 5 \times$ $5 \mathrm{~mm}$ ) that could be loaded with silicon powder (NBS 640c) to produce a user-defined distribution of scattering material along the length of the illuminated area. This was used to illustrate and test features of the partitioning algorithms along the illuminated area.

(b) A glass step wedge where the height of each step was $0.34 \mathrm{~mm}$ and where each step could be loaded with silicon powder. The area of each step was $\sim 2 \times 3 \mathrm{~mm}$. This provided an extended sample where each component step was displaced by a fixed amount from the centre of the diffractometer. This deliberate displacement error resulted in diffraction maximum shifts as 


$$
\Delta 2 \theta=-2 S \cos \theta / D
$$

where $\Delta 2 \theta$ is the variation of scattering angle, $D$ is the sampleto-detector distance and $S$ is the sample displacement relative to the instrument centre.

Thus this phantom represented a first approximation to a material with discrete changes in lattice parameter along the illuminated area.

(c) Homogeneous metallic thin films deposited upon glass slides. These were mounted within the diffractometer, initially with their surfaces parallel to the long axis of the incident beam (conventional setting). Subsequent data collections were undertaken with the samples rotated such that each end of the sample was displaced by the same amount (but one end a positive displacement and the other a negative displacement) from the instrument centre. The midpoint of the library sample remained on the instrument centre. Thus, as a result of the displacement gradient, the diffraction data would appear to derive from a continuously graded composition along the illuminated area. This gave diffraction maximum positional shifts analogous to the changes in lattice parameters expected

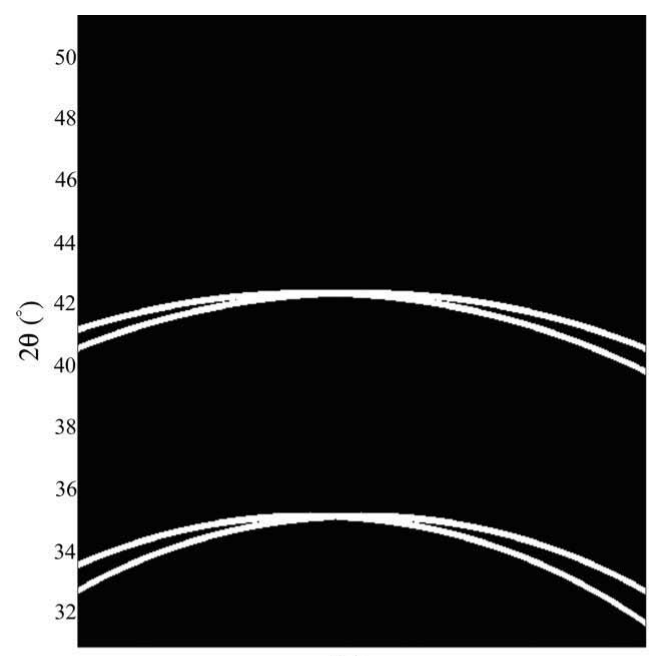

(a)

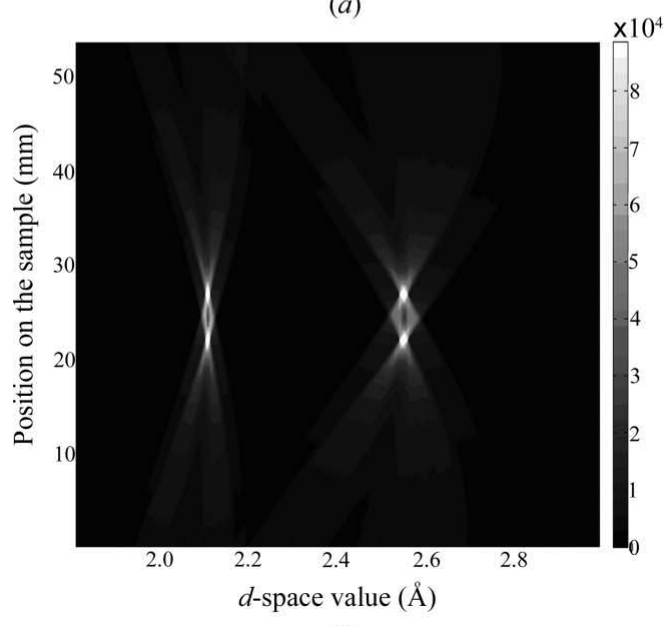

(b)

Figure 2

Simulated diffraction intensity distribution of two ideal compositional areas $(a)$ and the corresponding transformed distribution $(b)$. of samples with a changing compositional gradient obeying Vegard's law.

\section{Results and discussion}

In this section the data are presented in pairs in order that the scattering distributions can be easily compared with the corresponding transformed data.

\subsection{Simulations}

Bandit was employed to produce a number of simulated scattering distributions. These have the same number of pixels as the measured images $(1024 \times 1024)$. A simple example is shown in Fig. 1(a), which illustrates the intersection of Debye cones ( $d$ spacings of $2.4334,2.1075$ and $1.8859 \AA$ ) from a 'point', polycrystalline sample with a flat plate detector. This simulated distribution replicates diffraction from a cubic system with a lattice parameter of 4.216 (1) $\AA$. The corresponding transformed data are presented in Fig. 1(b), where the intensity distribution indicates, as expected, three discrete maxima at constant ' $y$ ' loci. As the simulated sample is a point source of scatter, there is no component of scattering along the

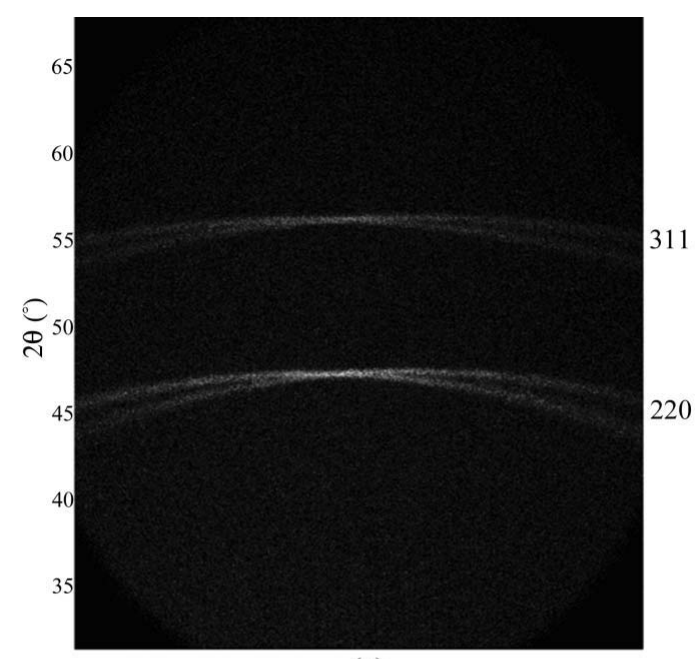

(a)

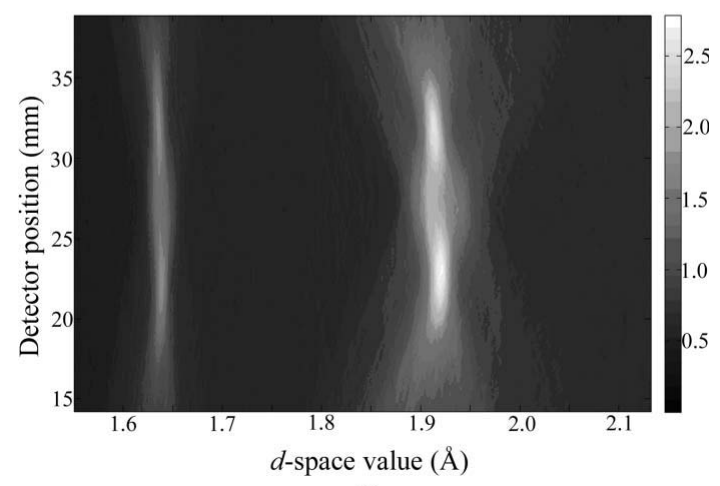

(b)

Figure 3

Experimentally produced diffraction intensity distribution from a twocomponent Si library $(a)$ and the corresponding transformed distribution (b). 
principal library axis, ' $y$ '. The $d$ spacings determined from the transformed data are $2.433,2.108$ and $1.886 \AA[a=$ 4.216 (2) $\AA$ ] , indicating the reliability of the transformation algorithm. Using repeated measurements, under these ideal conditions the minimum change detectable at a $d$ spacing of $2.5 \AA$ was determined to be $0.003 \AA$.

Fig. 2(a) is the simulated scattering distribution as would be measured from an ideal cubic material with diffraction maxima corresponding to $d$ spacings of 2.43 and $2.10 \AA[a=$ 4.204 (6) $\AA$ ]. In this case the model consists of two 'point' samples separated along the illuminated area by a distance of $4.8 \mathrm{~mm}$. The transformation algorithm produces four intensity maxima and clearly spatially separates the individual scattering centres (Fig. $2 b$ ). The $d$ spacings determined from the transformed data are 2.545 and $2.109 \AA$ [ $a=4.31$ (10) $\AA$ ], and the sample separation is measured to be $4.8 \mathrm{~mm}$. With these ideal conditions, the minimum step discernible in the $y$ axis was determined to be $2060 \mu \mathrm{m}$.

The linear array of wells was loaded with silicon powder (NBS 640c) by filling two of the wells separated by an unfilled well, i.e. two sources of diffraction with the same diffraction patterns but with the respective sample centres separated by

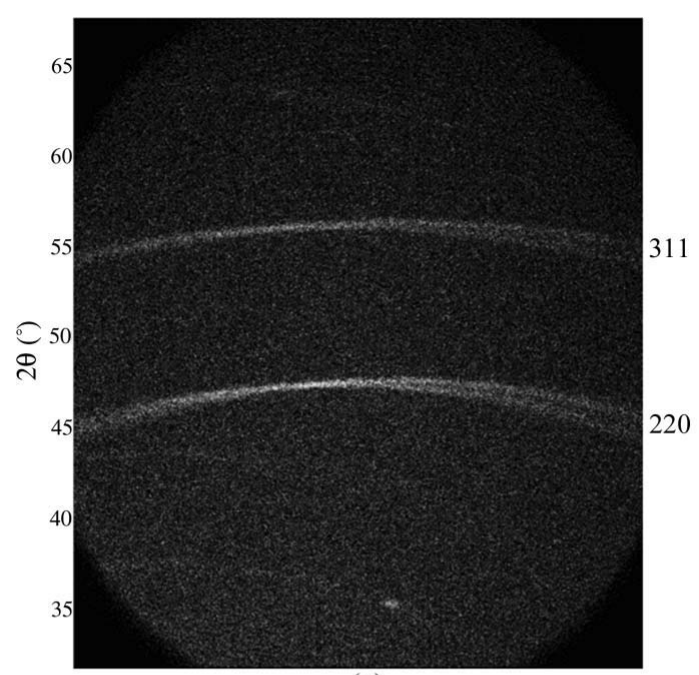

(a)

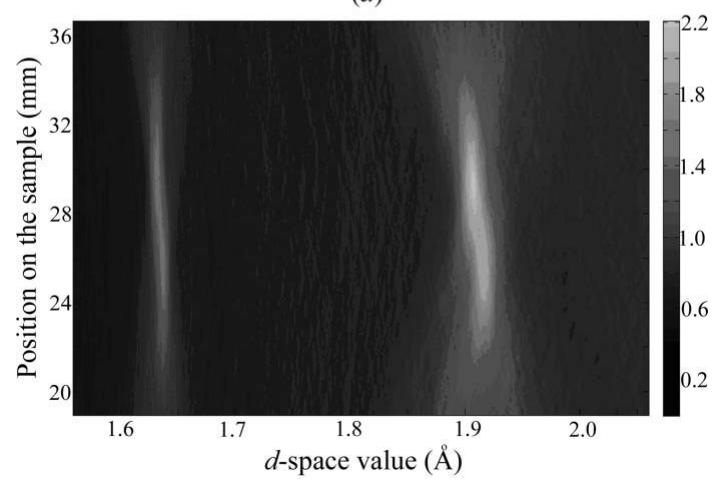

(b)

Figure 4

Experimentally produced diffraction intensity distribution of a twocomponent $\mathrm{Si}$ library with relative sample displacement between the areas $(a)$ and the corresponding transformed distribution $(b)$. $\sim 10.0$ (1) $\mathrm{mm}$ along the library principal axis. This was the experimental equivalent of the previously simulated case. The resulting diffraction pattern and transformed data are shown in Figs. 3(a) and 3(b), respectively. Compared with the equivalent simulation, the transformed maxima are extended in the ' $y$ ' direction. This is to be expected because of the finite well size $(5 \times 5 \mathrm{~mm})$ in contrast to the idealized simulation model possessing a minimal ' $y$ ' component. The sample separation was determined to be 9.89 (13) $\mathrm{mm}$. The $d$ spacings were determined to be $1.931 \AA$ for the 220 and $1.642 \AA$ for the 311 maxima $[a=5.45$ (1) $\AA$ ]. The NIST certified values for the corresponding maxima are $1.920 \AA$ for 220 and $1.640 \AA$ for 311 [ $a=5.435$ (6) $\AA$ from these maxima only] measured by conventional powder diffraction techniques.

An example of the scattering distribution from the glass step wedge phantom is shown in Fig. 4(a) where, for clarity, only two of the steps were loaded with silicon powder. The steps were separated by $4 \mathrm{~mm}$ along the illuminated area and were displaced in height by $0.34 \mathrm{~mm}$. The resulting transformed data are shown in Fig. 4(b), and from this intensity distribution, the distance between the steps was determined to be 3.9 (1) $\mathrm{mm}$. The transformed data were used to calculate

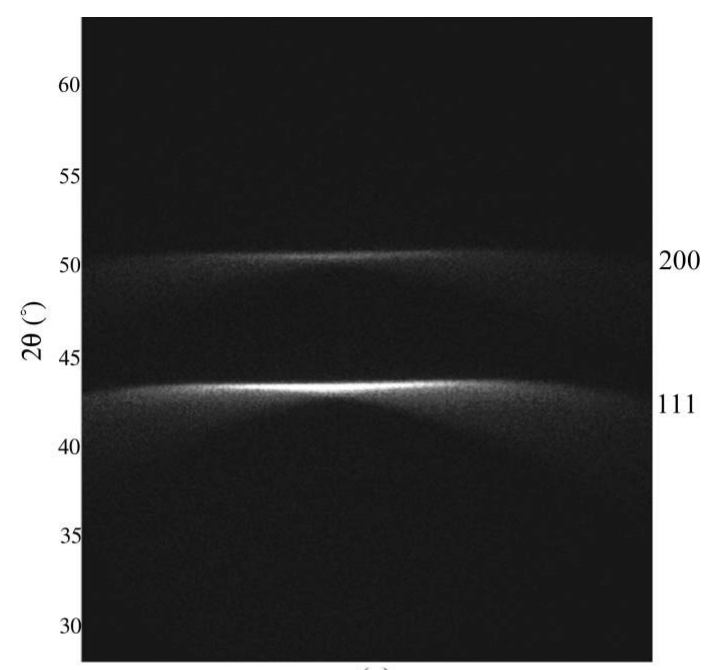

(a)

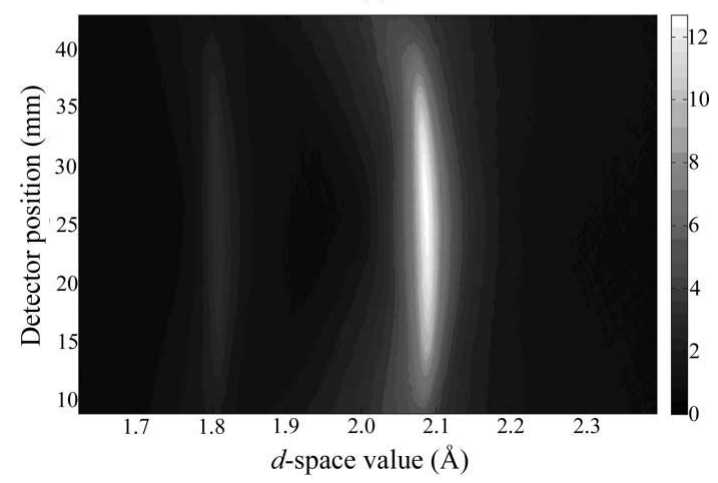

(b)

Figure 5

Experimentally produced diffraction intensity distribution of a homogenous copper thin film illuminated with an extended X-ray source $(a)$ and the corresponding transformed distribution $(b)$. 


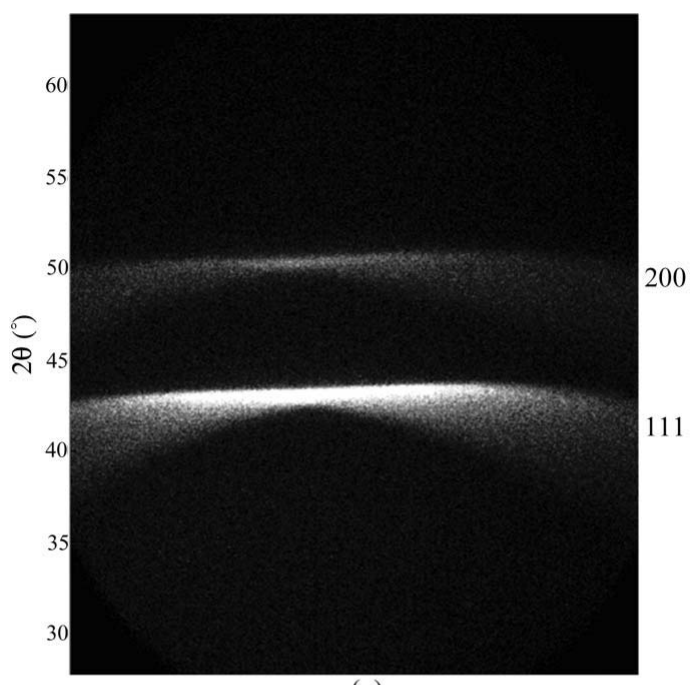

(a)

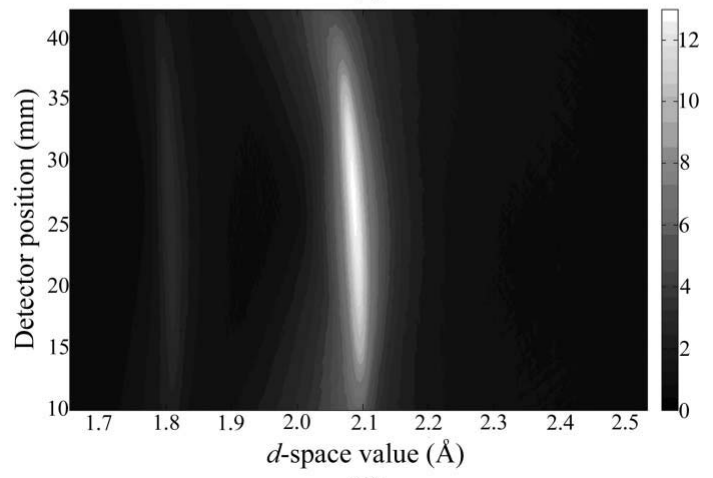

(b)

Figure 6

Experimental diffraction intensity distribution of a homogenous copper thin film tilted to simulate a compositionally graded material $(a)$ and the corresponding transformed distribution $(b)$.

differences in $d$ spacings from each of the steps of $0.008 \AA$ for 220 and $0.005 \AA$ for 311 . These are in good agreement with those calculated from equation (1).

The scattering data from a homogeneous $\mathrm{Cu}$ thin film deposited upon a glass slide and illuminated with the elongated source is shown in Fig. 5(a). The detector was positioned to receive scattering from the 111 and 200 crystal planes. The data transformed by Bandit are illustrated in Fig. 5(b), which indicates, as expected, two intensity maxima extended in the ' $y$ ' direction but each with a constant $d$ spacing corresponding to the 111 and 200 maxima of $\mathrm{Cu}$. Assuming a homogeneous film, any intensity variation at constant $d$ spacing would indicate variations in intensity along the interrogating X-ray beam, and this could be used subsequently as a correction factor.
To experimentally simulate scattering from a library possessing a continuous lattice parameter gradient, data from the copper thin film were collected following a rotation of the sample by $1^{\circ}$, thus creating a displacement error gradient. This was calculated to produce a change in $d$ spacing of 0.015 and $0.011 \AA$ across $24 \mathrm{~mm}$ for the 111 and 200 maxima, respectively. The consequent scattering data are presented in Fig. 6(a) and the corresponding transformed data in Fig. 6(b). The $d$-spacing changes measured from the transformed data were 0.015 and $0.010 \AA$ for 111 and 200, respectively. Thus we have been able to measure an apparent lattice parameter gradient of $9.911 \times 10^{-11}$ (equivalent to $9.911 \times$ $10^{-4} \AA \mathrm{mm}^{-1}$ ), which is probably better than that required for most materials.

\section{Conclusion}

In conclusion, we have been able to demonstrate how the use of an extended X-ray beam to illuminate compositional libraries has the potential to significantly reduce library interrogation times.

We have estimated the integrated volume separation for polycrystalline thin films to be approximately double that for the ideal case, i.e. $\sim 500 \mu \mathrm{m}$. Therefore it would be possible to extract measurements every $500 \mu \mathrm{m}$ along a library principal axis. Given our library length of $24 \mathrm{~mm}$, this approach would therefore reduce data collection times by a factor of almost 50 compared with an equivalent raster scan. Changes in lattice parameter along the library principal axis could be detected at a resolution $\left(\sim 10^{-3} \AA \mathrm{mm}^{-1}\right)$ that is suitable for most of the semiconductor materials that we are investigating.

\section{References}

Gorrie, C. W., Reese, M., Perkins, J. D., Alleman, J. L., Dabney, M. S., To, B., Ginley, D. S. \& Berry, J. J. (2008). 33rd IEEE Photovoltaic Specialists Conference, San Diego, CA, USA, 11-16 May.

Hest, M. F. A. M. van, Dabney, M. S., Peerkins, J. D. \& Ginley, D. S. (2006). Thin Solid Films, 496, 70-74.

Ohtani, M., Fukumura, T., Kawasaki, M., Omote, K., Kikuchi, T., Harada, J., Ohtomo, A., Lippmaa, M., Ohnishi, T., Komiyama, D., Takahashi, R., Matsumoto, Y. \& Koinuma, H. (2001). Appl. Phys. Lett. 79, 3594-3596.

Takeuchi, I., Lippmaa, M. \& Matsumoto, Y. (2006). MRS Bull. 31, 999-1002.

Thelakkat, M., Schmitz, C., Neuber, C. \& Schmidt, H.-W. (2004). Macromol. Rapid Commun. 25, 4-223.

Yanase, I., Ohtaki, T. \& Watanabe, M. (2002). Solid State Ionics, 151, 189-190.

Zhao, J.-C. (2006). Prog. Mater. Sci. 51, 557-631. 